\title{
Book Review - Buchbesprechumg - Livre nouuveau
}

H. Vagtborg (Ed.): The Baboon in Medical Research, Volume П. University of Texas Press, Austin/London 1967. XIII + 908 pp. Price: \$ 15.00.

Im November 1965 fand das zweite «Pavían-Symposium» der Southwest Foundation for Research and Education in San Antonio, Texas, statt. Von den 165 Teilnehmern werden in dem von H. Vagtborg sorgfältig redigierten und mit einem Vorwort versehenen Band 67

Originalarbeiten vorgelegt. Mitarbeiter der Southwest Foundation sind mit 24 Arbeiten beteiligt, für eine Reihe von weiteren wurden Paviane der Foundation verwendet. So enthält dieser zweite Band (Vol. I siehe Folia primat. 4: 398, 1966) wieder einen grossen Teil der Ergebnisse, die in den Labors der Foundation in San Antonio und Kenya gewonnen wurden.

Den Schwerpunkt bilden nicht, wie der Titel vermuten lassen könnte, nur den Mediziner interessierende Arbeiten. Selbst unter den zehn Beiträgen des Kapitels «Experimental Medicine» sind drei von allgemeinem Interesse. Die vielen neuen Informationen über die Biologie freilebender und gefangener Paviane in den übrigen fünf Kapiteln machen das Buch für jeden, der sich für Primaten interessiert, zu einer reichen Fundgrube, ganz gleich, ob er stammesgeschichtliche Fragen bearbei-tet und die Daten zum Vergleich heranziehen will, ob er Versuche an lebenden Pavianen ausführen oder die beschriebenen Techniken bei anderen Primaten ver-wenden will. Die Überschriften der Kapitel deuten das breite Spektrum an: «Taxonomy and Colony Management» (mit 7 Beiträgen), «Anatomy and Embryology» (10), «Pathology and Physiology» (19), «Biochemistry» (10) und «Microbiology» (11). Mehrere Beiträge hätten anders eingeordnet werden können. Es ist unmöglich, in diesem Rahmen jede Arbeit angemessen zu besprechen.

Die Systematik, Klassifikation und Nomenklatur der Savannen-Paviane be-spricht W. C. Osman Hill (S. 3). Viele Fragen müssen offen bleiben, beispielsweise ist die Subspezies-Gliederung der

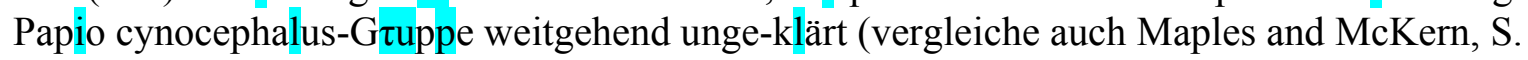
13), was wegen der vielfach auftre-tenden Unterschiede innerhalb einzelner und zwischen verschiedenen Populationen (z.B. Serumproteine Kitchin et al., S.637) bedauerlieh ist. Vorläufig ist ein Pavian dieser Gruppe durch eine genaue geographische Herkunftsangabe am besten charakterisiert.

Jolly (S. 23) bespricht die Phylogenese der «baboons». Unglücklich erscheint dem Referenten die Definition eines «baboon» als eines Angehörigen der Cercopithecidae mit einem «lndex of Arboreality» von weniger als 30. Der Index wird nicht klar definiert. Dschelada und Husarenaffe (Erythrocebus) sind für Jolly «baboons», der Mandrill nicht. (In alien übrigen Arbeiten wird oft von «the baboon» gesproehen. Gemeint ist immer ein Angehöriger der Papio cynocep $7 / 8 \alpha /$ usGruppe). Am Skelett rezenter Cercopithecidae beschreibt Jolly Merkmale der Anpassung an terrestrische Lebensweise und diskutiert dann, welche fossilen Vertreter der Familie diese Merkmale haben. Sie müssen voneinander unabhängig mehrfach auf-getreten sein.

Hummer (S.51), Hendrickx and Kriewaldt (S.69), Creager and Switzer (S.85), Hughes (S.99) und Hsiung et al. (S.797) besprechen Einfuhr, Quarantäne, Haltung, Zucht und Aufzucht.

244 Book Review - Buchbesprechung - Livre nouveau 
Die Beiträge über die normale Anatomie sind besonders wertvoll, weil sie die individuelle Variabilität ausreichend berücksichtigen. Untersuchern von Weich-teilen stand bisher selten umfangreiches Material aus einzelnen Wildpopulationen zur Verfügung. (Boyden, S. 117: Ductus choledochus und Ductus pancreatici; McCoy et al., S. 151: Arteria carotis externa; Herrmann et al., S.535: Herz; Swindler, S. 133: Zähne; Benton, S. 201: Lumbalwirbelsäule, Becken und Muscu-lus erector trunci vieler Primatengattungen).

Einen Schwerpunkt bilden mehrere Beiträge über Fortpflanzungsbiologie und Ontogenese der Paviane. Axelrod (S. 633) beschreibt die Testosteronsynthese im Hoden, Chiarelli (S. 271) die Chromosomen in der Meiose, Katzberg (S. 217) die Eientwicklung und (S.235) die mikroskopische Anatomie von Vagina und Uterus; Hendrickx (S.437) den Menstruationszyklus. Hervorgehoben werden soil die Beschreibung der äusseren Körperform von 45 Embryonen im Alter von 22 Tagen bis 15 Wochen durch Hendrickx (S. 283). Die einzelnen Entwicklungsstadien wur-den durch Kaiserschnitt gewonnen (Technik: Claborn et al. S. 825), das Konzep-tionsdatum war meist bekannt. Rhesusaffe und Mensch sind die einzigen Primaten, von denen bisher solche Serien vorlagen. - Beiträge zur Kenntnis der postnatalen Entwicklung von Skelett und Gebiss bringen Reed (S.181) und Snow (S. 187).

Von den physiologischen, biochemischen, mikrobiologischen und parasitolo-gischen Arbeiten werden diejenigen das weiteste Interesse finden, die erstmals in dieser Vollständigkeit an einer grossen Zahl von Individuen gewonnene «Normal-werte» zusammenstellen. Fife (S.493) berechnet Herzminutenvolumen und Durch-blutung einzelner Organe. Immer wieder überrascht die technische Perfektion. Wer hätte sich vor ein paar Jahren vorstellen können, dass man die Nierendurchblutung eines am Nordhang des Kilimandjaro in Freiheit lebenden Pavians kontinuierlich misst bis zu dem Augenblick, in dem ihn ein Leopard tötet (van Citters et al., S.473)? de la Peña and Goldzieher (S. 379) bringen in übersichtlichen Tabellen Differentialblutbilder und eine Unmenge Angaben über Plasma- und Urinbestand-teile gesunder Paviane, die ergänzt werden durch elektrophoretische Analysen der Plasmaproteine von Groover and Stout (S.605) und Beobachtungen über die Blutgerinnung von Hampton and Matthews (S. 659). Fünf Arbeiten über «Micro-biological Parameters of the Baboon» (Kalter, Al-Doory, Kuntz and Pinker-ton, S. 715-773) berichten systematisch über Bakterien, Pilze, Viren und Parasiten, die bei Pavianen in Kenya und in San Antonio gefunden wurden.

Auch der Ethologe findet in dem Band vieles: Ohne direkte Kontakte wird der Menstruationszyklus weiblicher Paviane durch die Anwesenheit eines erwach-senen Männchens im gleichen Versuchsraum stark verändert (Smith et al., S.621). Die Körpertemperatur gesunder Paviane, deren tageszeitliche Schwankungen hier erstmals telemetrisch aufgezeichnet werden, ändert sich schon bei geringer Erregung stark (Hendrickx et al., S.419). Die Farbbevorzugung kann durch leichte Erkran-kungen beeinflusst werden (Groover et al., S.617).

Aus den Gebieten der Pathophysiologie und Pathochemie sei auf jeweils mehrere Arbeiten über Atherosklerose und Organtransplantationen hingewiesen.

Die Diskussionen zu den einzelnen Vorträgen sind alle im Wortlaut wieder-gegeben und enthalten viele wichtige Ergänzungen.

Die Ausstattung des Bandes ist hervorragend, der Preis niedrig. Hoffentlich folgen weitere Berichte über Symposien der Southwest Foundation for Research and Education in gleicher Weise! Hans-Jürg Kuhn, Frankfurt a. M. 